\title{
Complete reversibility of central pontine myelinolysis not associated with hyponatremia
}

\author{
Ebru Erbayat ${ }^{1}$, Pravin George ${ }^{2}$, Aarti Sarwal ${ }^{3}$ and Christopher R. Newey ${ }^{*}$ \\ *Correspondence: crnewey@gmail.com

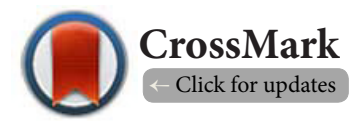 \\ 'Department of Neurology, Cleveland Clinic Foundation, Cleveland, Ohio, USA. \\ 2Department of Neurology, Johns Hopkins University, Baltimore, Maryland, USA. \\ ${ }^{3}$ Wake Forest University, Baptist Health System, Winston-Salem, North Carolina, USA. \\ ${ }^{4}$ Department of Neurology, University of Missouri, Columbia, Missouri, USA.
}

\begin{abstract}
Background: Central pontine myelinolysis (CPM) is a demyelinating disorder most frequently described in alcoholism and malnourishment after rapid correction of hyponatremia affecting the pontine base. We present a case of reversible CPM as demonstrated on neuroimaging and at autopsy in a patient with recent upper respiratory infection and vitamin B12 deficiency.

Case persentation: Patient presented with lower extremity weakness after upper respiratory infection. She had a normal sodium level, but found to have low vitamin B12 level. MRI of the brain showed restricted diffusion in the pontine basis. She was given empiric prednisone for myopathic symptoms and vitamin B12 supplementation for her low level. She made full clinical recovery by 5 months. She developed pneumonia 6 months after initial presentation. Repeat MRI showed resolution of the hyperintensity in the pontine basis. Her respiratory status worsened, and she died. At autopsy, there was no evidence of demyelination in the pontine basis.

Conclusion: The importance of this case is that in CPM not associated with hyponatremia the radiologicalpathological correlation of osmotic demyelination can be completely reversible.
\end{abstract}

Keywords: Central pontine myelinolysis, vitamin B12 deficiency, non-hyponatremia

\section{Introduction}

Central pontine myelinolysis (CPM) is a demyelinating disorder that classically affects basis pontis and particularly occurs in chronic alcoholism, liver failure, and chronic malnourishment [1]. Although vitamin B12 deficiency does not cause CPM, it is a surrogate marker for malnourishment [2]. We present a case of reversible CPM in a patient with history of gastric bypass presumed to be related to vitamin B12 deficiency.

\section{Case presentation}

A fifty-five year old female with previous history of gastric bypass for morbid obesity presented with new onset proximal lower extremity weakness after a 4-week history of upper respiratory symptoms. On admission, her vitals were stable, and she was on room air on the regular nursing floor. An inflammatory myopathy was suspected with an electromyography (EMG) showing mild to moderate myopathic motor unit potentials but an unremarkable muscle biopsy. Magnetic resonance imaging (MRI) of the craniospinal axis revealed hyperintensity in the basis pontis consistent with acute/subacute central pontine myelinolysis with restricted diffusion (Figure 1A). Further diagnostic work up revealed normal metabolic profile, including a normal sodium level without any significant change during hospitalization, or history of abnormal polydypsia. She was found to have hypoalbuminemia with an albumin of $1.9 \mathrm{~g} / \mathrm{dL}$ and protein of $4.1 \mathrm{~g} / \mathrm{dL}$ though both were normal 6 weeks prior. Serum Vitamin B12 levels were noted to be low $(177 \mathrm{pg} / \mathrm{mL}$; normal 221-700pg/mL) with an MMA level $397 \mathrm{mmol} / \mathrm{L}$ (normal 79-376). She was given empiric prednisone for myopathic symptoms and vitamin B12 supplementation and made full recovery from weakness by five months since presentation. Incidentally, she was admitted to the medical intensive care unit for a protracted respiratory illness 6 months later. Brain MRI was obtained as part of a work up for a persistent encephalopathy given previous history of CPM. The hyperintensity on MRI had resolved (Figure 1B). She eventually had a complicated medical course 


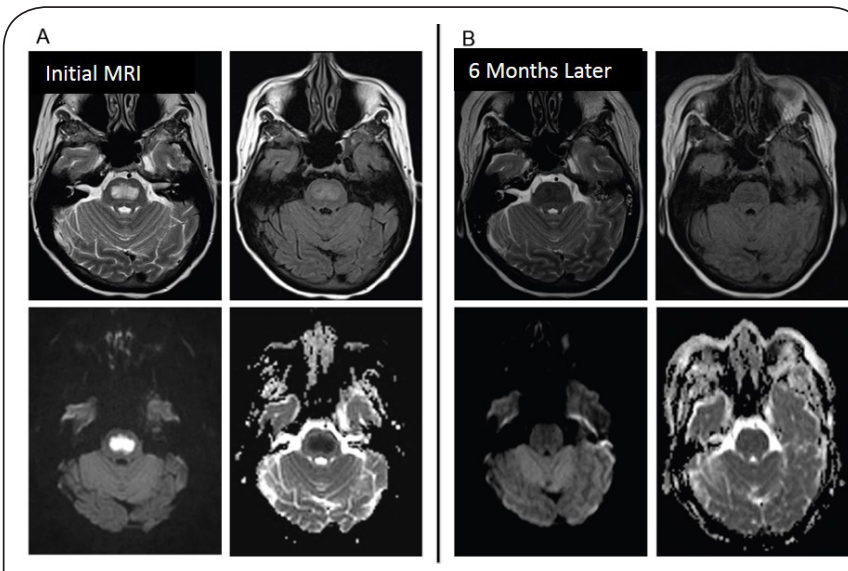

Figure 1. Magnetic Resonance Imaging at CPM diagnosis and six months later.

Brain MRI. (A) T2, T1, DWI and ADC sequences (clockwise from left upper corner) showing acute/subacute central pontine myelinolysis affecting basis pontis. (B) The central pontine myelinolysis resolved on 6-month follow up MRI along improvement in clinical recovery.

of pneumonia and expired. An autopsy was performed, and it did not show any evidence of demyelination or abnormal brainstem pathology (Figure 2).

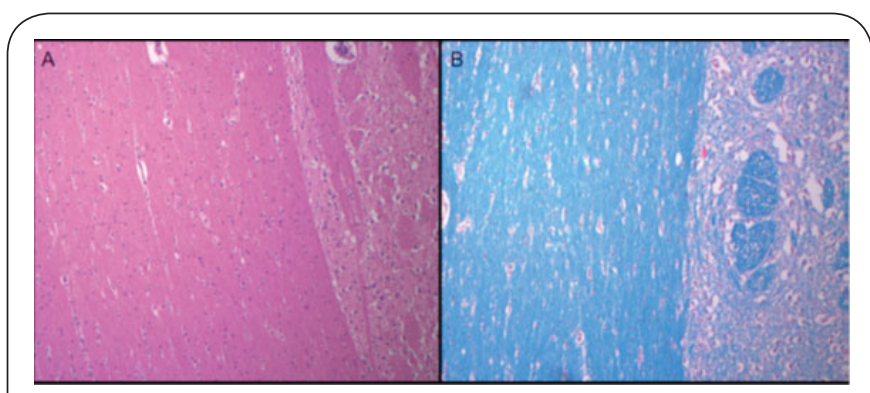

Figure 2. Hematoxylin-Eosin (H\&E) and Luxol Fast Blue (LFB) stain of pons.

Autopsy specimen of the pons showing no histological evidence of myelinolysiswith both H\&E (A) and Luxol Fast Blue (B) Stains.

\section{Discussion}

Excitotoxicity, as demonstrated on diffusion-weighted imaging sequence of brain MRI, is typically seen in cases of osmotic demyelination [3]. It likely reflects destruction of myelin sheaths at susceptible locations, such as the pontine basis [4]. The exact pathophysiology is not known. It may be a result of impaired glutamate receptor function by the development of organic osmolytes $[4,5]$. The development of organic osmolytes makes myelin susceptible to damage with rapid changes in tonicity such as during rapid correction of chronic hyponatremia $[4,5]$. A systematic review of the literature found that the most common predisposing etiology of osmotic demyelination is hyponatremia [6]. Importantly, autoimmune etiologies that are responsive to steroid therapy are becoming recognized [7]. Over half of all patients with osmotic demyelination not associated with liver failure eventually have a favorable recovery even with severe, initial neurological presentation [6].

This case suggests an uncommon etiology for osmotic demyelination in the setting of hypoalbuminemia and an upper respiratory infection that responded to the combination of vitamin B12 supplementation and steroid therapy. It highlights that in non-hyponatremia CPM the radiological-pathological correlate can be completely reversible. When an uncommon etiology for CPM is suspected, exhaustive workup, including evaluation for autoimmune etiologies, should occur and guide management prior to prognostication. These patients may be responsive to steroids.

\section{Competing interests}

The authors declare that they have no competing interests.

Authors' contributions

\begin{tabular}{|l|c|c|c|c|}
\hline Authors' contributions & EE & PG & AS & CRN \\
\hline Research concept and design & $\checkmark$ & $\checkmark$ & $\checkmark$ & $\checkmark$ \\
\hline Collection and/or assembly of data & $\checkmark$ & $\checkmark$ & $\checkmark$ & $\checkmark$ \\
\hline Data analysis and interpretation & $\checkmark$ & $\checkmark$ & $\checkmark$ & $\checkmark$ \\
\hline Writing the article & -- & -- & -- & $\checkmark$ \\
\hline Critical revision of the article & $\checkmark$ & -- & -- & $\checkmark$ \\
\hline Final approval of article & $\checkmark$ & $\checkmark$ & $\checkmark$ & $\checkmark$ \\
\hline Statistical analysis & -- & -- & -- & -- \\
\hline
\end{tabular}

\section{Acknowledgement}

The authors would like to acknowledge Dr. Richard Prayson, M.D., for the pathological images.

\section{Publication history}

Editors: Raffaele Izzo, Federico II University Hospital, Italy. Mahdi Najafi, Tehran University of Medical Sciences, Iran. Received: 29-Feb-2016 Final Revised: 24-Mar-2016 Accepted: 05-Apr-2016 Published: 14-Apr-2016

\section{References}

1. Norenberg MD. Central pontine myelinolysis: historical and mechanistic considerations. Metab Brain Dis. 2010; 25:97-106. | Article | PubMed

2. Nagaishi $A$, Yukitake $M$, Eriguchi $M$ and Kuroda Y. [A case of alcoholic with vitamin B12 deficiency presenting central pontine and extrapontine myelinolysis on MRI]. Rinsho Shinkeigaku. 2007; 47:173-6. | Article | PubMed

3. Cramer SC, Stegbauer KC, Schneider A, Mukai J and Maravilla KR. Decreased diffusion in central pontine myelinolysis. AJNR Am J Neuroradiol. 2001; 22:1476-9. | Article | PubMed

4. Moritani T, Smoker WR, Sato $Y$, Numaguchi $Y$ and Westesson PL. Diffusion-weighted imaging of acute excitotoxic brain injury. AJNR Am J Neuroradiol. 2005; 26:216-28. | Article | PubMed

5. Lien YH. Role of organic osmolytes in myelinolysis. A topographic study in rats after rapid correction of hyponatremia. J Clin Invest. 1995; 95:1579-86. | Article | PubMed Abstract | PubMed FullText

6. Singh TD, Fugate JE and Rabinstein AA. Central pontine and extrapontine 
Erbayat et al. Journal of Medical Disorders 2016,

myelinolysis: a systematic review. Eur J Neurol. 2014; 21:1443-50. |

Article | PubMed

7. Menon B, Bedi SS and Rao GU. Combined central and peripheral demyelination. J Neurosci Rural Pract. 2014; 5:78-80. | Article | PubMed Abstract | PubMed FullText

\section{Citation:}

Erbayat E, George P, Sarwal A and Newey CR. Complete reversibility of central pontine myelinolysis not associated with hyponatremia. J Med Disord. 2016; 4:1.

http://dx.doi.org/10.7243/2053-3659-4-1 